RESEARCH ARTICLE

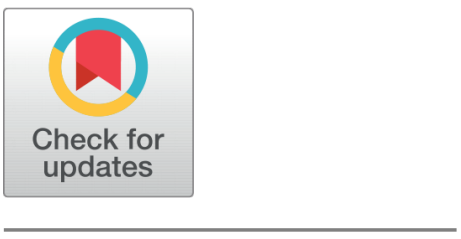

OPEN ACCESS

Received: 09.08.2020

Accepted: 13.10 .2020

Published: 06.11.2020

Editor: Dr. Natarajan Gajendran

Citation: Albay EM, Alambra RVE, Masiong DVM, Imperial DM (2020) Sustaining the efficiency in research of an academic unit in a State University in Northern Philippines: Results from a data envelopment analysis study. Indian Journal of Science and Technology 13(39): 4084-4091. https://doi.org/ 10.17485/IJST/v13i39.1618

* Corresponding author.

ealbay@dmmmsu.edu.ph

Funding: Don Mariano Marcos Memorial State University

Competing Interests: None

Copyright: (c) 2020 Albay et al. This is an open access article distributed under the terms of the Creative Commons Attribution License, which permits unrestricted use, distribution, and reproduction in any medium, provided the original author and source are credited.

Published By Indian Society for Education and Environment (iSee)

ISSN

Print: 0974-6846

Electronic: 0974-5645

\section{Sustaining the efficiency in research of an academic unit in a State University in Northern Philippines: Results from a data envelopment analysis study}

\author{
Eduard M Albay ${ }^{1 *}$, Ralph Vincent E Alambra1 , Dan Vergel M Masiong1 , \\ Delia M Imperial'1
}

1 Faculty Members, Don Mariano Marcos Memorial State University, Philippines

\section{Abstract}

Background/Objectives: The general aim of this study is to determine the performance efficiency in research of the four departments of the College of Arts and Sciences in Don Mariano Marcos Memorial State University (DMMMSU), Philippines during the years 2014, 2015, and 2016. It also determined the: (1) performance efficiency level of the departments in research; (2) peer groups and weights of the departments; and (3) virtual inputs and virtual outputs or potential improvements of the departments. Methods/Statistical analysis: This study employed the descriptive evaluative research design. Primarily, the study is a document-based analysis study. Data in research gathered from the existing research-related documents of the four departments of the college were analyzed using Data Envelopment Analysis (DEA) Solver. Findings: Findings of the study showed that: $50 \%$ of the departments are fully efficient while the other $50 \%$ are inefficient in research. The fully efficient departments, BPSD and MADD, serve as models for improvement in research of the inefficient departments, LD and HSSD, to be in the efficient frontier. The BPSD and MADD serve as peers for the inefficient HSSD and LD, respectively, with different weights to consider. Fully efficient departments do not have virtual inputs and virtual outputs. Only HSSD and LD posted virtual inputs and virtual outputs in the research input and output indicators to become fully efficient. Novelty/Applications: Based on the results, a Sustainability Training Series in Research was developed to strengthen the involvement of faculty members in research of all the departments in the College of Arts and Sciences and to sustain the performance in research of the college in the entire university. The said training series was implemented in the last quarter of 2016 up to the present.

Keywords: Data envelopment analysis; performance efficiency; research; sustainability training series 


\section{Introduction}

In the Philippines, colleges and universities, which are referred to as higher education institutions (HEIs), are considered one of the major entities that generate, transmit, disseminate, and apply knowledge which are considered vital components of the nation's research and innovation system ${ }^{(1)}$. Thus, every faculty member is expected to be the primary producers of knowledge through the conduct of relevant researches in the university ${ }^{(2)}$.

The knowledge generated from researches and applied to various fields of interests serves as lifeblood of any knowledgebased economy. It helps develop inquisitiveness, critical and independent thinking. Further, research-based knowledge advances the "boundaries of knowledge and understanding"(1).

In light of this reality, the Philippines' Republic Act No. 7722 or known as the "Higher Education Act of 1994" mandates the Commission on Higher Education (CHED) to promote, direct and support HEIs in performing their research and instruction functions to produce high-quality research that improves learning and contributes to national development. The Act requires CHED to perform its functions related to research which are as follows: a) formulate and recommend development plans, policies, priorities and programs on research; b) recommend to the executive and legislative branches, priorities and grants on higher education and research; c) develop criteria for allocating additional resources such as research and program development grants, scholarships, and other similar programs; and d) direct or redirect purposive research by institutions of higher learning to meet the needs of agro-industrialization and development.

The CHED has been consistently showing fervent and zealous determination in pushing HEIs in the country to establish and maintain an excellent research orientation and culture. In compliance to RA 7722, CHED formulated the National Higher Education Research Agenda (NEHRA) in 1996 which provides research policies and directions among HEIs in the country. The main objectives of NEHRA are a) to improve research capability and productivity of HEIs, b) to generate leading edge knowledge and technologies, and c) to promote and facilitate the dissemination and utilization of research outputs ${ }^{(3)}$.

The Don Mariano Marcos Memorial State University (DMMMSU), one of the Level IV state universities and colleges (SUCs) in the country, continue to lead in the generation of relevant knowledge to achieve a peaceful, equitable, self-sufficient and ecologically-balanced society through its commitment to research ${ }^{(4)}$.

The goal of DMMMSU Research Unit is to generate and verify appropriate technologies and information in response to the priority needs of its various clienteles in instruction and extension. To accomplish this, research endeavor shall focus on relevant concerns in education, agriculture, agroforestry, fisheries, trade and industry, health and nutrition, local government administration, energy and socio-economic which are adaptable and responsive to the immediate needs of its clientele thereby having a peaceful, just, self-sufficient and ecologically-balanced society in support to the manufacturing, industry and service sectors $^{(4)}$.

As a consequence of the university's commitment to research, DMMMSU is among the Level IV SUCs in the country in $2014^{(5)}$. Further, the university is one of the top 22 leading SUCs in the country and one among the only 22 SUCs belonging to the top performers in research and development. This recognition is the result of the concerted efforts of the university's different colleges and institutes in instruction, research, extension, and production ${ }^{(6)}$.

In $2010,{ }^{(7)}$ conducted a study on the performance efficiency of the 16 colleges and institutes, generally referred to as academic units, of DMMMSU. One of the indicators is the units' contribution to research. The study involved the following variables: number of on-going researches, number of research staff/personnel, number of linkages in research, number of completed researches, number of published researches, and number of researches presented in local to international conferences. The study found out that 15 out of 16 colleges and institutes are fully efficient in research. Among the fully efficient colleges is the College of Arts and Sciences (CAS) in the South La union Campus (SLUC).

The College of Arts and Sciences is DMMMSU's second best academic unit in 2014. Then, the college was adjudged as the Best Academic Unit for two consecutive years, in 2015 and 2016. Among the areas evaluated in the ranking of colleges and institutes is the unit's involvement in research. The CAS has four academic departments - the Biological and Physical Sciences Department (BPSD), the Humanities and Social Sciences Department (HSSD), the Languages Department (LD), and the Mathematics and Allied Disciplines Department (MADD).

Ranking of academic units in the university based on their accomplishments in various aspects, including research, is considered as the best practice of the university since 2012. The performance of one college in all the indicators is influenced by the individual contributions of each faculty member. Based on evaluation records, the colleges in the university indicate close scores in all the parameters except for research and extension.

Although the College of Arts and Sciences has been the top academic unit in the whole DMMMSU system in 2015 and 2016, not all its departments are contributing equally to the college's accomplishments in research which are the primary bases for evaluating its performance in the said parameter. Because every department contributes significantly to the overall performance of the college, the current study aimed to determine the efficiency in research of the four departments of the College of Arts and 
Sciences based on existing research-related documents in 2014, 2015 and 2016 using Data Envelopment Analysis. Specifically, this study determined the performance efficiency level in research of the departments of CAS using DEA, identified the peer groups (reference or model for improvement) and weights (percentage to be adopted) in research of the inefficient departments of CAS, and identified the virtual inputs or virtual outputs (potential improvements) in research of the departments of CAS to be in the efficient frontier.

The results of this study served as input in the development of a Sustainability Training Series in Research for the College of Arts and Sciences. The training series aimed to strengthen the participation in research of the faculty members from each department of the college, hence contributing significantly to the college intention to sustain its performance as the top performing college in the whole DMMMSU system by strengthening its accomplishments in the different activities in research such as submission of research proposals for approval and inclusion to the annual research work and financial plan, conduct and completion of approved research studies, high rate of faculty involvement in research as determined by the ration of faculty members with approved researches to the total number of faculty members of an academic unit, presentation of research outputs to scientific conferences, publication of research articles to at least local journals, obtaining research-related awards, publication of college journal and newsletter, conduct of research-related capability-building or enhancement activities, and other relevant endeavors.

This study offered a pro-active approach in encouraging faculty members of an academic unit to be involved in creating an excellent research culture and maintaining the efficiency in research of an academic unit by doing an internal evaluation of the unit's strengths and weaknesses in the said field. By scientifically considering the strong points and weak points of the academic unit, the research personnel can develop a capability enhancement intervention that aimed at achieving the desired level of involvement and accomplishments of faculty members in research, thus, contributing to the overall performance of the academic unit.

\section{Framework of the study}

The onset of globalization demanded the critical attention to research in order to generate knowledge and discover new strategies ${ }^{(8)}$. HEIs do not only produce graduates. More importantly, they create knowledge ${ }^{(9)}$. Towards this end, the Commission on Higher Education (CHED) in 1994 was mandated to ensure and protect the advancement of learning in higher education institutions through productive and useful researches, pursuant to Republic Act No. $7722^{(10)}$.

Thus, universities include in their functions the conduct of research which primarily pushes the generation of knowledge and relevant technologies.

Philippine colleges and universities, as centers of knowledge production and generation, play a critical role in the country's national research and development projects. Thus, research capacities increasingly receive recognition as one important indicator in assessing the performance efficiency of HEIs in the country. Consequently, promoting research performance and striving for research excellence has become a prominent goal to be attained by the HEIs in the country.

DMMMSU supports researches that lead to the innovation, integration and circulation of new knowledge which are contributory to the attainment of its philosophy, vision, mission, and goal. At the same time, the university recognizes that research is fundamentally bind to the quality of its teaching, programs, and outreach or community extension projects. To advocate this worthwhile endeavour and to preserve integrity in the research and scholarly activities of its manpower, DMMMSU has established a number of policies that deliver, institute, and standardize research practices at the university. The level to which the various colleges and institutes of the university adheres to the policies on research determine their efficiency.

The current study is based on the belief that every college or institute in the university must maintain an excellent research culture and environment in order to advance knowledge, and apply new knowledge to improve the quality of human life and respond effectively to the challenges of globalization hence contributing to the overall performance of DMMMSU in the State Universities and Colleges (SUC) Levelling.

Therefore, this study endeavored to determine the efficiency in research of the four departments of the College of Arts and Sciences namely Biological and Physical Sciences Department (BPSD), Humanities and Social Sciences Department (HSSD), Languages Department (LD) and Mathematics and Allied Disciplines Department (MADD). Numerical data were taken from the existing research-related documents at the CAS Research Office covering the years 2014, 2015, and 2016.

From the results, a Sustainability Training Series in Research for the CAS was developed to strengthen faculty members' engagement and accomplishments in research and to sustain the performance efficiency of the college in terms of research in the entire university. 


\section{Materials and Methods}

This study employed the descriptive evaluative research design. Primarily, the study is a document-based analysis study. The four departments of the College of Arts and Sciences - BPSD, HSSD, LD, and MADD, served as the decision-making units (DMUs) or respondents of this study. Necessary data for the study were collected from the existing research-related documents of the four academic departments that are available at the CAS Research Office. A structured instrument which purely asks for research-related quantitative data was utilized.

The variables of this study were categorized as input variables and output variables. To ensure uniformity and balance in terms of indicators, the variables were derived from indicators in the evaluation of academic units of DMMMSU with respect to research. The study considered only those indicators that pertain to the individual accomplishment of faculty members.

The input variables of the study are the following: a) number of faculty members, and b) number of on-going researches. For the output variables, these include the a) number of faculty involved in research, a) number of completed researches, b) number of research presentations, and c) number of researches published in journals. For the research presentation and publication, points were allocated depending on the scope of the scientific conference or the journal. The highest point of five (5) was given to each presentation or publication at the international level, 3 points for national or regional level, and 1 point for local presentation or publication.

The study used frequency and means to determine the numerical data for each input and output variable. The Multi-Stage Input-Oriented Constant Returns-to-Scale Model through DEA add-ins in MS Excel was utilized in the analysis of data. The Multi-Stage Input-Oriented Constant Returns-to-Scale Mode is appropriate to use in determining the desired weight and efficiency of a DMU to enhance its efficiency by minimizing its inputs while producing the projected amount of outputs ${ }^{(11)}$.

\section{Results and Discussion}

\subsection{Performance efficiency in research}

The means and total frequencies of the data gathered for each of the input and output variables in research from the four departments of the College of Arts and Sciences from 2014 to 2016 were determined and were plugged-in into the DEA Solver which is an add-in component of the Microsoft Excel. The data were analyzed by the DEA Solver to determine the performance efficiency scores in research of the departments.

Table 1 presents the input and output scores, and efficiency level in research of the BPSD, HSSD, LD, and MADD.

The table shows that the departments have varying input and output data. As to input indicators, HSSD and MADD have the greatest and least number of faculty members, respectively. On the other hand, LD posted the highest number of on-going researches with an average of 13 distinct researches annually.

Table 1. Efficiency level in research of the departments in the CAS

\begin{tabular}{lllll}
\hline \multirow{2}{*}{ Indicators } & \multicolumn{3}{c}{ Decision-Making Units } \\
\cline { 2 - 5 } & BPSD & HSSD & LD & MADD \\
\hline Input & 19 & 20 & 17 & 15 \\
\hline 1. Number of faculty & 7 & 7 & 12 & 11 \\
2. Number of on-going researches & & 9 & 1 & 15 \\
\hline Output & 12 & 2 & 10 & 7 \\
\hline 1. Number of faculty involved in research & 3 & 2 & 33 \\
2. Number of completed researches & 11 & 12 & 51 \\
3. Number of research presentations & 54 & $0.75^{*}$ & $0.71^{*}$ \\
4. Number of research articles published & $1.00^{* * *}$ & $1.00^{* * *}$ & \\
\hline Efficiency Score & & & & \\
\hline
\end{tabular}

${ }^{* * *}$ Fullyefficient ${ }^{* *}$ WeakEfficient ${ }^{*}$ Inefficient

In terms of the output variable, $100 \%$ of the MADD faculty members are involved in research undertakings while there is only $45 \%$ or nine faculty members involved in research from the HSSD. Of the 11 average ongoing researches from the MADD, a total of seven or 64\% was completed from 2014 to 2016. Though LD has the highest number of ongoing researches, it recorded the lowest number of completed research with only one completed research study in a period of three years. Further, presentation of research outputs to scientific conferences and publication of research articles to refereed journals are noted as 
strengths of the BPSD and MADD. For the research presentation, the BPSD posted an average of 11 points while 33 points for the MADD. For the publication of research articles, the BPSD and MADD recorded an average points of 54 and 51, respectively.

The performance of the four departments in research can be generalized in terms of their efficiency scores. It can be noted from the table that research is a strength of the BPSD and MADD as reflected in their efficiency scores of 1.00, described as fully efficient. On the other hand, HSSD and LD were found to be inefficient in research in the included years of evaluation as their input and output scores deliver efficiency scores of 0.75 and 0.71 , respectively.

Results of the analyses reflect that the fully efficient departments, BPSD and MADD, are committed in achieving excellent research performance by providing the faculty members with an effective research capacity building management system. Their dedication and active involvement in research endeavours are reflected in the number of on-going and completed researches, and number of faculty members who are involved in research undertakings. These were supported by the publication of their outputs in different local and international journals, and presentation to various local and international conferences. These accomplishments contributed significantly to the departments' fully efficient performances in research.

The BPSD and MADD, though operating with full efficiency, are encouraged to continuously promote a research-based environment in their respective department to sustain their performance efficiency.

On the other hand, the weak efficient performances of HSSD and LD may have been resulted from the very high frequencies in the input indicators but low output scores or accomplishments. To become fully efficient, the two departments are recommended to perform necessary improvements in their research operations as reflected in the succeeding discussion of results. They may consider a substantial percentage of the best practices of the other two fully efficient departments. Further discussion on the potential improvement of the HSSD and LD are presented in the peers and weights, and virtual input and virtual output.

\subsection{Peers and weights}

One of the advantages of DEA is its capacity to provide ways for weak efficient or inefficient DMUs to become fully efficient. DEA identifies peers or references for potential improvement for inefficient DMUs. These peers are fully efficient. Aside from this, DEA also provides data on the amount or percentage that the weak efficient or inefficient DMUs should consider from their peer/s. This is known as the weights. Fully efficient DMUs do not have peers and weights as they are already operating in the efficiency frontier, thus, no radial movement is necessary to perform.

The peers and weights of each inefficient department that are necessary to bring them to the efficient frontier are shown in Table 2.

Table 2. Peers and weights of the departments

\begin{tabular}{lll}
\hline Department & Peers & Weights \\
\hline BPSD & BPSD & 1.00 \\
HSSD & BPSD & 0.75 \\
LD & MADD & 0.80 \\
MADD & MADD & 1.00 \\
\hline
\end{tabular}

It could be gleaned from the table that the fully efficient BPSD and MADD have their own department as their peers since they are already fully efficient in research. This means that they do not need peers as their references, since no radial movement or actions for improvement are required. Thus, they serve as references (peers) for potential improvements by considering a percentage (weights) of their best practices in research by the inefficient departments.

To become fully efficient, HSSD needs to examine the best practices in research of the BPSD being its peer. Moreover, HSSD is recommended to adopt 75\% of the practices of BPSD. On the other hand, it is recommended for the LD to benchmark on the best practices of the MADD being its peer. Further, LD needs to consider implementing in their department some preeminent practices in the research operations of the MADD to pull its performance efficiency level to full efficiency.

Given these data on peers and weights, the inefficient departments may initiate a cross-analysis of the current practices of their fully efficient counterparts to evaluate and identify the root cause of their inefficiencies in research. There is a necessity for the HSSD and LD to heighten the involvement of their faculty members in research. In so doing, this may result to a higher data in the output indicators particularly on the number of completed research, and presentation and publication of research outputs. Specific targets or projected input and output data for the HSSD and LD are presented in the discussion for virtual input and virtual output. 


\subsection{Virtual input and virtual output}

The identification of DMUs with best operating practices may lead to an improvement in the performance of all units being compared, especially those which are inefficient. The DMUs that appear efficient based on the DEA Solver definitely represent conditions of comparison and examples to be imitated by the other units. In this way, a unit might appear efficient by purposely attributing a reasonable weight only to a certain set of inputs and outputs.

In cases that some DMUs appeared off the efficient frontier, the Peers and Weights show the needed percentage of each inefficient DMU from its peers to be in the efficient frontier. This potential improvement of the inefficient DMUs is shown in Virtual Inputs/Outputs (IO) sheet.

Virtual inputs and virtual outputs provide information on the relative importance that each DMU attributes to each individual input and output, for the purpose of maximizing its own efficiency score, and at the same time improving the efficiency of those units which are weak efficient or inefficient. Thus, this allows each unit to identify and highlight their specific competencies or strengths, at the same time, their weaknesses ${ }^{(12)}$.

Table 3 shows the IO sheet of the four departments of CAS which indicates their individual potential improvement in research. For each department, the figures in the first column in the input and output variables denote the department's score, while the second column contains the target or projected scores of the department to become fully efficient. The virtual input suggests a considerable amount of decrease (-) in the initial input scores while the virtual output recommends some increase $(+)$ in the initial output scores.

Table 3. Virtual input and virtual output of the departments

\begin{tabular}{|c|c|c|c|c|}
\hline \multirow{2}{*}{ Indicators } & \multicolumn{4}{|c|}{ Projected Input (-) and Output (+) } \\
\hline & BPSD & HSSD & LD & MADD \\
\hline \multicolumn{5}{|l|}{ Virtual Input } \\
\hline 1. Number of faculty & $19(19)$ & $20(14)$ & $17(12)$ & $15(15)$ \\
\hline 2. Number of on-going researches & $7(7)$ & $7(5)$ & $13(8)$ & $11(11)$ \\
\hline \multicolumn{5}{|l|}{ Virtual Output } \\
\hline 1. Number of faculty involved in research & $12(12)$ & $9(9)$ & $12(12)$ & $15(15)$ \\
\hline 2. Number of completed researches & $3(2)$ & $2(2)$ & $1(5)$ & $7(7)$ \\
\hline 3. Number of research presentations & $11(11)$ & $2(8)$ & $10(26)$ & $33(33)$ \\
\hline 4. Number of research articles published & $54(54)$ & $12(41)$ & $27(41)$ & $51(51)$ \\
\hline
\end{tabular}

It can be noted from the table that the target or projected input and output values for the BPSD and MADD are the same as their scores. This is primarily the consequence of their fully efficient performance level in research. However, the HSSD and LD need to do necessary adjustments in their input and output scores to consequently obtain fully efficient scores. Although the HSSD posted the highest average number of faculty members and the $L D$ has the greatest average number of ongoing researches, these figures do not guarantee a fully efficient performance level for both the departments. This is primarily due to the fact that the numbers do not sufficiently commensurate to their scores in the output variables.

For both HSSD and LD, DEA results suggest that in order that the number of completed research matches with the corresponding number of ongoing researches, they need to decrease the number of researches being conducted from 7 to 5 for the HSSD and from 13 to 8 for the LD. However, practically speaking, the result suggests that faculty members from the HSSD and LD must follow the indicated schedule of implementation of their research studies. Ongoing researches from these departments must finish the conduct of these researches based on the approved work and financial plan. It is recommended therefore that the research facilitators of the HSSD and LD, in coordination with the college research coordinator, must constantly monitor the progress of the individual researches in order that faculty members will be given necessary support and assistance to meet the expected completion of their research studies.

Further, results reflect that the HSSD and LD need to strengthen their involvement in research in terms of presentation and publication. For the HSSD, it needs to increase its data on presentation to $300 \%$ or an equivalent increase of 8 points in research presentations from its current data on said indicator. For the publication of research outputs, the department is highly encouraged to attain a score of 41 or an equivalent increase of $341 \%$ in order to become fully efficient. For the LD, it needs to accumulate a total points of 26 and 41 for research presentation and publication, respectively. The points can be earned depending on the number of research articles or research presentations and the level of the journal where these were published or the level of the scientific conferences where the research papers were presented. 


\subsection{Sustainability training series in research}

The results of the analyses made on the data gathered as presented in the above discussion served as concrete bases in developing the Sustainability Training Series in Research for all the faculty members of the four departments of the College of Arts and Sciences. The training series is composed of ten (10) distinct series. The first nine series cover topics on writing a research proposal, data management and analysis, interpreting the results of the analyses, writing the terminal report, and dissemination of research results through presentation and publication. The last series is a special capability building program that is in a form of a writeshop which can be scheduled anytime, or as the need arises to facilitate faculty researchers in preparing their research monitoring reports.

The implementation of the Sustainability Training Series in Research in the College of Arts and Sciences in the last quarter of 2016 facilitated the college in strengthening the involvement and engagement of faculty members of the college in the different research-related activities of the university. The training series was considered as one best practice of the college in research since its implementation. As results, the percentage of faculty members involved in research through the number of faculty members with approved research proposal increased. The completion rate of approved researches also increased. The college consistently recorded significant numbers of research outputs presented in various conferences and research articles published in different journals. Consequently, the College of Arts and Sciences sustained its rank as the best performing academic unit from 2017 to 2019. To date, the CAS has been the best performing academic unit in the entire DMMMSU system for five consecutive years, 2015 to 2019. With the continuous implementation of the sustainability training series in research in the college, the CAS hopes to receive the same recognition for 2020 .

\section{Conclusion}

The study aimed to determine the efficiency level in research of the four departments of the College of Arts and Sciences using the Data Envelopment Analysis Solver in MS Excel. The results of the study based on the data gathered from the identified respondents or decision-making units indicate that the departments, BPSD and MADD, are fully efficient and have the best research practices in the CAS while the HSSD and LD are inefficient in research. To become fully efficient with respect to the input and output variables included in this study, HSSD and LD need to look into the operations and best practices in research of BPSD and MADD, respectively, by considering their projected inputs and outputs values.

The results of the analysis provided by DEA Solver in MS Excel offered baseline information for the researchers to develop a Sustainability Training Series in Research for the College. The implementation of the Sustainability Training Series in Research in the College of Arts and Sciences strengthened the involvement and engagement of faculty members of the in the different research-related activities as mandated by the university. Consequently, the College of Arts and Sciences has been the best performing academic unit for five consecutive years now, 2015 to 2019, in the whole DMMMSU community.

This research study had offered a practical and concrete application of the abstract concepts of linear programming to improving the efficiency of an academic institution by performing an internal evaluation of its strengths and weaknesses, and carefully considering the results as bases for crafting a strategic plan for the purpose of sustaining the institution's efficiency level.

For future research studies, it would be very interesting to include other parameters and variables necessary to fully evaluate the efficiency level of institutions in a particular aspect.

\section{Acknowledgement}

The authors thank the administrators of the Don Mariano Marcos Memorial State University for providing opportunities and funding support for completion of this research study.

Declaration of ownership: This report is the original work of the authors.

Ethical clearance: This study was approved by the institution.

\section{References}

1) Angeles EY. National Higher Education Research Agenda 2009-2018. 2010. Available from: www.ched.gov.ph.

2) Clemeña RM, Acosta SA. Developing research culture in Philippine higher education institutions: Perspectives of University faculty. 2007. Available from: http://unesdoc.unesco.org/images/0015/001578/157869e.pdf.

3) Bustamante C. Higher Education Research in the Philippines: Policies and Prospects. 2018. Available from: 10.1007/978-981-10-4989-7_16.

4) DMMMSU Research and Extension Manual. 2017.

5) CHED. SUC Level. 2014. Available from: http://ched.gov.ph/wp-content/uploads/2014/12/SUC-Level.pdf. 
6) Baldemor M. Efficiency of Local Government Units in North Western Philippines as to the Attainment of the Millennium Development Goals. Data Science: Journal of Computing and Applied Informatics. 2017;1(1):21-29. Available from: https://dx.doi.org/10.32734/jocai.v1.i1-64.

7) Baldemor MR. Performance Efficiency of DMMMSU Colleges and Institutes: A Data Envelopment Analysis (DEA) Study. College of Graduate Studies, DMMMSU-SLUC. 2018.

8) Jr MAF. Predictors of research dissemination and utilization in state higher education institutions in Region IV, Philippines. Liceo Journal of Higher Education Research. 2010;6(2). Available from: https://dx.doi.org/10.7828/ljher.v6i2.79.

9) Ogena OB. PNU Research Agenda 2013-2015. 2013. Available from: http://www.pnu.edu.ph/wp-content/uploads/2014/08/PNU-Research.

10) CHED. Republic Act 7722 - Higher Education Act of 1994. 1994. Available from: http://www.gov.ph/downloads/1994/05may/19940518-RA-07722-FVR. pdf.

11) Baldemor MR. Time Series and Data Envelopment Analysis on the Performance Efficiency of DMMMSU-South La Union Campus. International Journal of Scientific Engineering and Research (IJSER). 2016;4(4):5-10.

12) Albay EM. Performance Efficiency of College of Computer Science of State Universities and Colleges in Region I: A Data Envelopment Analysis Study. International Journal of Scientific \& Engineering Research. 2014;5(7):1360-1371. 